\title{
DIE TRANSZENDENZ DER GEWALT \\ IM ANGESICHT DER WENDE - THOMAS HETTCHES NOX UND DIE BESONDERE KONSTELLATION DER POSTMODERNE
}

\begin{abstract}
The transcendence of violence in the face of German Reunification - Thomas Hettches Nox and the particular configuration of postmodernity

The year 1989 not only marks the end of the Cold War and the German reunification, it also initiates a specific period in which post-modern philosophy reached its peak. The political and social situation in the Occident seemed to be so stable that scientists as well as the general public started to speak of the "end of history". This was accompanied by a certain feeling of loss of reality, intensified by the development of electronic media. This little Belle Époque of the $90 \mathrm{~s}$ was the breeding ground for a peculiar understanding of violence that was expressed in popular culture or even in such phenomena as extreme sports. The violence was in fact seen as one of the few ways to transcend the boundaries of the world not only broadcasted but also created by the mass media. With the spirit of the times corresponds the novel Nox by Thomas Hettche. The text, considered as one of the first novels of German Reunification, brings up and connects two transitions - the one, regional, from the divided to the reunified Germany and the other, related to all spheres of reality, from modernity to post-modernity. In both processes, the infamous, autotelic violence emerges (Reemtsma), this time, in the constellation of postmodernity, under the guise of transcendence.
\end{abstract}

IST ES GOTTLOS, WENN JEDER SELBST BESTIMMT, WIE ER LEBEN WILL. WER GIBT EINEM DAS RECHT AUF SELBSTBESTIMMUNG, WENN NICHT GOTT, UND GOTT MACHT DIE GESETZE. UND JEDER IST SEIN EIGENER GOTT, UND GOTT HAT DEN URKNALL GEHÖRT. ER WEISS, WAS DAS BEDEUTET.

Tim Staffel, Terrordrom ${ }^{1}$

\section{Die Belle Époque der 90er Jahre}

Die Nacht vom 9. zum 10. November 1989, die in die Geschichte als die Nacht des Mauerfalls eingegangen ist, markiert einen Wendepunkt nicht nur im geschicht-

\footnotetext{
${ }^{1}$ Tim Staffel: Terrordrom, München 2000, S. 28.
} 
lichen, sondern auch im symbolischen Sinne. Die Wende bedeutet das Ende des Kalten Krieges und die deutsche Wiedervereinigung, sie leitet aber auch eine besondere Zeitperiode ein, in der die postmoderne Weltanschauung ihren Höhepunkt erreichte. Der Zusammenbruch des kommunistischen Systems schien die These von der Überlegenheit der liberalen Demokratie zu bestätigen, vielmehr das liberaldemokratische System wurde als die endgültige, den höchstmöglichen Grad der sozialen Ordnung sichernde Regierungsform betrachtet, was in der, genauso populären wie missverstandenen, Theorie vom Ende der Geschichte des US-amerikanischen Politikwissenschaftlers Francis Fukuyama mündete. ${ }^{2}$ Mit der zunehmenden Stabilität der politischen und sozialen Lage in der westlichen Welt ging aber ein gewisses Gefühl der Entwirklichung ${ }^{3}$ einher. Die Omnipräsenz der Massenmedien und der von ihnen nicht nur übertragenen, sondern kreierten Wirklichkeit bestätigte die These, die Marshall McLuhan bereits Ende der 50er Jahre aufgestellt hat, nämlich, dass das Medium selbst die Botschaft bildet. ${ }^{4}$ Die Grenze zwischen medialer Wirklichkeitsrepräsentation und -reproduktion schien verwischt zu sein. Der französische Philosoph und Medientheoretiker Jean Baudrillard sprach von der Welt der Simulacra ${ }^{5}$, deren Höhepunkt der erste Golfkrieg gebildet habe. ${ }^{6}$ Die politische und

\footnotetext{
${ }^{2}$ Fukuyama postulierte keine Einstellung des geschichtlichen Prozesses, was seiner Theorie - nach gängiger Meinung - unterstellt wurde. Mit dem Ende der Geschichte meinte er die allgemeine Annahme der liberalen Demokratie als die endgültige Regierungsform, was den höchsten Grad der sozialen Ordnung sichern sollte. Nach der Annahme des liberal-demokratischen, d.h. des bestmöglichen Systems wäre keine weitere Entwicklung im sozialen und politischen Bereich möglich. Der historische Prozess gelangte somit zur Vollendung, was jedoch das weitere Stattfinden von historisch relevanten Ereignissen nicht ausschließt. Francis Fukuyama: The End of History and the Last Man, New York 2006.

${ }^{3}$ Der Begriff wird hier nicht im Kontext der Philosophie von Max Scheler oder Sigmund Freuds Psychoanalyse, sondern vor allem als ein medienästhetisches Phänomen verstanden, das auf der subjektiven Auflösung der Wirklichkeit und der Wirksamkeit von Verhältnissen der Wirklichkeit beruht. Die medial vermittelte Wirklichkeit kann von dem Beobachter als unwirksam wahrgenommen werden, die virtuelle Realität kann dagegen durch ein durch technische Mittel bewirktes Feedback als wirksam wahrgenommen werden. Mit der Spannung zwischen Realität und Virtualität, Repräsentation und Simulation geht ein Gefühl der Unsicherheit und des Zweifels an der Wirkung der im gegebenen Augenblick vorhandenen (realen oder virtuellen) Wirklichkeit einher. Diese Empfindung kann als Entwirklichung bezeichnet werden. Vgl. Peter Matussek: Real Virtuality - Die Wahrheit der Entwirklichung, in: Space and Truth - Raum und Wahrheit, hrsg. von Thea Breszek, Wolfgang Greisenegger, Lawrence Wallen, Zürich 2009, S. 206-215.

${ }^{4}$ Marshall McLuhan: The Medium Is the Message, in: ders.: Understanding Media: The Extensions of Man, Cambridge 1994, S. 7-21.

${ }^{5}$ Simulacrum bedeutet nach Baudrillard ein referenzloses Zeichen. Jean Baudrillard: Simulacres et Simulation, Paris 1981.

${ }^{6}$ Baudrillard wurde durch seine Feststellung bekannt, dass der Golfkrieg eigentlich nicht stattgefunden hat. Gemeint ist hier, dass die mediale Repräsentation des Krieges die tatsächlichen Kriegshandlungen überdeckt und entstellt hat. Die angebliche Übertragung authentischer Ereignisse hat sich in eine Reproduktion von gewalttätigen Kriegsbildern, in ein virtuelles Kriegsspiel mit wirklichen Opfern verwandelt, das keineswegs der Nachrichtenversorgung, sondern vor allem der Öffentlichkeitsarbeit dienen sollte. Vgl. Jean Baudrillard: Amerika, Berlin 2006. Zur Problematik des ersten Golfkrieges und seiner medialen Repräsentation siehe auch Paul Virilio: Krieg und Fernsehen, Frankfurt/Main 1997.
} 
ökonomische Lage löste ein geistiges Klima der entrealisierten Medienwelt, des materiellen und symbolischen Überschusses aus. Damit ging aber ein diffuses Gefühl der Ohnmacht einher, in die die Hochkultur des Westens geriet, nachdem sie den Zustand des Endes der Geschichte erreicht hat, d.h. ihren durch den demokratisch-liberalen Kapitalismus symbolisierten Höhepunkt. Da sich die kapitalistische Demokratie als die bestmögliche gesellschaftliche Ordnung erwiesen hat ${ }^{7}$, sei die weitere Entwicklung der sozialen Verhältnisse nicht nur unnötig, sondern auch unmöglich, der Fortschritt sei ausgeschlossen, es bleibe nur übrig, das Leben in dem perfekten System zu genießen. Gleichzeitig war aber der Zeitgeist dieser Epoche durch die Vorahnung einer nahenden Katastrophe, durch die Überzeugung, man lebe in der Endzeit, geprägt, was ihren Ausdruck in solchen kulturellen Phänomenen fand, wie der Popliteratur, die Mitte der 90er Jahre in Deutschland ihre Renaissance erlebte. Die selbst ernannten Dandys und dekadenten Autoren des „popkulturellen Quintetts ${ }^{\text {“8 }}$ beschworen ein neues Fin-de-Siècle herauf. Das Zeitgeistgefühl fand Resonanz auch in Texten anderer Autoren, die dem Quintett zwar nicht angehörten, aber deren Werke manchmal der Popliteratur zugerechnet werden. ${ }^{9}$ Die Pop-Texte spiegeln ein gewisses Gefühl der Trägheit, des Driftens wider, das als Folge der Fragmentarisierung der Gesellschaft in der flüchtigen ${ }^{10}$ Postmoderne betrachtet werden kann. In der atomisierten Welt ist es nicht nur unmöglich, auf die sozialen Verhältnisse Einfluss zu nehmen, sondern es hätte auch keinen Sinn, denn alle Bestrebungen, die Welt zu ändern und sich auf irgendeine Art und Weise zu engagieren, stellen nur ein Hindernis dar am Weg zum einzigen Ziel des postmodernen Konsumenten: dem hedonistischen Sammeln von Eindrücken. ${ }^{11}$ Das System des liberalen

\footnotetext{
${ }^{7}$ Darauf soll ihr Sieg im „Kampf“ mit dem Sozialismus und Kommunismus hindeuten. Vgl. z.B. Robert Heilbroner: The Triumph of Capitalism, in: The New Yorker, 23.01.1989, S. 98. Auf die Alternativlosigkeit der liberalen Demokratie gründet sich auch die oft missverstandene Theorie Fukuyamas.

${ }^{8}$ Diese Bezeichnung geht auf die von Joachim Bessing bearbeitete und unter dem Titel Tristesse Royale: Das popkulturelle Quintett veröffentlichte Aufzeichnung der Gespräche zwischen ihm und anderen Pop-Schriftstellern - Benjamin von Stuckrad-Barre, Christian Kracht, Eckhart Nickel und Alexander von Schönburg zurück. Joachim Bessing, u.a.: Tristesse Royale: Das popkulturelle Quintett, Berlin 1999.

${ }^{9}$ Wie z.B. Sibylle Berg, Clemens Mayer oder Tim Staffel, um hier nur die repräsentativsten Autoren zu nennen.

${ }^{10}$ Die Bezeichnung „flüchtige Moderne“ wird zum zentralen Begriff in der Theorie eines der bekanntesten Postmoderne-Forschers Zygmunt Bauman. Die „flüchtige“ Moderne, d.h. die Postmoderne, unterscheide sich von der „schweren“ Moderne u.a. durch die Erosion sozialer Beziehungen und durch das Verschwinden zentralistischer Machtinstanzen. Siehe dazu: Zygmunt Bauman: Flaneure, Spieler und Touristen. Essays zu postmodernen Lebensformen, Hamburg 1997, S. 119-169 und Zygmunt Bauman: Flüchtige Moderne, Frankfurt/Main 2003.

${ }^{11}$ Die Konsumenten- und Spielerrollen seien, so Bauman, die wichtigsten Muster der Individualitätsbildung unter postmodernen Bedingungen. $\mathrm{Zu}$ den Kennzeichen dieser Muster gehören u.a. der Nachdruck auf die Erfahrungssammlung durch ein Individuum, Spontaneität und Mobilität oder Fitness
} 
Kapitalismus verlangt Flexibilität und Anpassungsfähigkeit, Eigenschaften also, die jede Bindung an Familie, Heimat, oder sogar an die eigene feste Identität ausschließen. Der Verzicht auf jeden festen Halt in der Welt hat jedoch seinen Preis: den postmodernen Menschen durchdringen Gefühle der Orientierungslosigkeit und Verlorenheit. Die populären und Pop-Autoren greifen das Thema in ihren Texten auf, indem sie unter dem Deckmantel des konsumverherrlichenden Marktenthusiasmus den Leerlauf der postmodernen Gesellschaft bloßlegen. Der deutsche Schriftsteller Mario Wirz bringt in einer seiner Erzählungen aus der Sammlung Umarmungen am Ende der Nacht diese Problematik griffig auf den Punkt ${ }^{12}$ und deutet zugleich einen möglichen Ausweg aus dem Zustand des Identitätsmangels an:

Namenlos will ich sein und ohne Geschichte. Ohne Vergangenheit und ohne Zukunft. Nur Haut und Augenblick. ${ }^{13}$

Die kleine Passage weist einerseits auf die Probleme hin, die das postmoderne Individuum mit der Bestimmung der eigenen Identität haben kann, andererseits macht sie auf die Grenze der Atomisierung aufmerksam, die zugleich den letzten Halt, den letzten festen Punkt in der Welt der Flüchtigkeit bilden kann, nämlich auf den Körper. Auch in anderen Texten lässt sich eine Bestätigung für das nahe Verhältnis zwischen dem Zeitgeist des postmodernen Fin-de-Siècle und dem Körperdiskurs feststellen. Zum Beispiel äußert sich der Hauptprotagonist in Tim Staffels Roman Rauhfaser über postmoderne Ängste und Methoden und Wege, sie zu bewältigen, etwas ausführlicher als der Erzähler im Text von Mario Wirz:

Ich fühlte mich von nichts betroffen, nur der immerselben grenzenlosen Ohnmacht ausgesetzt, die mich im Wortgewäsch einer blindverzerrten Welt, an die man glauben sollte, aufrieb. Aber auch ich wollte mich retten. Ich entschied, Vergangenheit und Zukunft auszublenden, suchte nach der Lücke einer gedankenlosen Gegenwart. Meine Finger tasteten nicht mehr nach der Welt; ich fand die Nische, die mich von ihr trennte - ich zog nach Fehrbellin, in die Nähe des Flugplatzes. Springen. Fliegen. Einmal im Monat kam Primavesi mich besuchen. [...] Er wunderte sich über meine Strategie der Angstbewältigung, aber entscheidend ist nicht der Fall, entscheidend ist die Landung. [...] Es ging nicht um den Freifall, die Kontakte, Bewegungen und Rotationen in der Luft, das Schweben am Schirm. Es ging mir um den Augenblick des Absprungs, um die Sekunden, sobald man sich abdrückt, losspringt, nach vorn überkippt. ${ }^{14}$

im Sinne der Fähigkeit, einen wachsenden Umfang neuer Erfahrungen aufzunehmen. Bauman: Flaneure, Spieler und Touristen, S. 247-257.

${ }^{12}$ Auf diese Passage verweist Thomas Jung in seinem Beitrag zur populären und Popliteratur, der eine gute Einführung in die Thematik bietet. Siehe Thomas Jung: Trash, Cash oder Chaos? Populäre deutschsprachige Literatur seit der Wende und die sogenannte Popliteratur, in: Alles nur Pop? Anmerkungen zur populären und Pop-Literatur seit 1990, hrsg. von Thomas Jung, Frankfurt/Main 2002, S. 15-27.

\footnotetext{
${ }^{13}$ Mario Wirz: Umarmungen am Ende der Nacht, Berlin 2004, S. 9.

${ }^{14}$ Tim Staffel: Rauhfaser, Frankfurt/Main 2002, S. 137-138.
} 
Das Hier und Heute des eigenen Leibs ist die letzte und, genau genommen, einzige Instanz, auf die sich der postmoderne Mensch verlassen kann. Der Körper wird zum unabdingbaren Medium des Wissens- und Erfahrungstransfers. Die einzige wahre, unverfälschte und nicht medial entstellte Erfahrung ist die Erfahrung des eigenen Körpers. Die einzige Möglichkeit, sich über die eigene Identität Klarheit zu verschaffen, besteht auf der Beschränkung der eigenen Person auf den Körper und dessen somatische Reaktionen. Mit dieser Verengung der Perspektive geht jedoch eine wichtige Gefahr einher, denn die Reduzierung auf den Körper lässt nur die Aufnahme von rein physischen Reizen zu und schließt jegliche geistige Wahrnehmung aus. Anders gesagt - um von etwas Kenntnis zu bekommen, muss man damit in physischen Kontakt treten; je heftiger der Kontakt ist, desto intensiver ist die Wahrnehmung. Die Möglichkeit einer Eskalation, die in Gewalt mündet, liegt hier auf der Hand. Nach dem deutschen Philologen und Literaturwissenschaftler Jan Philipp Reemtsma bedeute das Phänomen der Gewalt zunächst physische Gewalt und diese impliziere immer eine Reduktion des Gewaltopfers auf seinen Körper, wobei diese Reduktion keine sich nur auf die Körperlichkeit beschränkende Wahrnehmung, sondern wortwörtlich die Herabsetzung des Betroffenen zu einem Stück Fleisch bilde. ${ }^{15}$ In diesem Sinne verwandelt sich die postmoderne Identitätssuche, die darauf beruht, dass sich das Individuum auf einen Eindrücke sammelnden Körper selbst beschränkt, in eine Autoaggression, auch wenn die gegen sich selbst gerichtete Gewalt nur in Form einer Androhung besteht. Das trifft im Fall des Protagonisten aus Staffels Roman zu: er bewältigt seine Ängste vor der flüchtigen Wirklichkeit, indem er sich selbst zu einem in der Luft rotierenden und schwebenden Körper, der außerdem bei jedem Sprung der Gefahr des gewaltsamen Todes ausgesetzt ist, reduziert. Die einzigen Grenzen, die Mitglieder der flüchtigen Gesellschaft der Postmoderne erfahren können, sind die Grenzen ihrer eigenen Körper und diese sind nur durch Schmerz wahrnehmbar. In diesem Sinne ähnelt die Identitätssuche des postmodernen Individuums den Versuchen eines Psychotikers, sich durch Selbstverstümmelung der Körpergrenzen zu vergewissern. ${ }^{16}$

So bildete die kleine Belle Époque der 90er Jahre den Nährboden für ein eigentümliches Verständnis von Körperlichkeit und Gewalt, das nicht nur in zahlreichen kulturellen Texten, wie in den angeführten Werken der Pop-Autoren, sondern auch in solchen Phänomenen wie Extremsportarten ${ }^{17}$ oder Subkulturen des Modern Pri-

\footnotetext{
${ }^{15}$ Jan Philipp Reemtsma: Vertrauen und Gewalt: Versuch über eine besondere Konstellation der Moderne, Hamburg 2008, S. 124-125.

${ }^{16}$ Ebd., S. 104.

${ }^{17}$ Wie sie übrigens in einigen Texten - wie Staffels Rauhfaser - thematisiert wurden. Die Extremsportarten scheinen sich gut in die postmoderne Mentalität einzufügen. Laut Bauman bilde die Fitness das Hauptmodell der Anpassung an die Erwartungen der postmodernen Gesellschaft (im Gegensatz zur Gesundheit in der Gesellschaft der Moderne). Fitness wird hier verstanden als das körperliche und geistige Vermögen des Individuums, einen wachsenden Umfang neuer Reize aufzunehmen. Bauman: Flaneure, Spieler und Touristen, S. 250. In diesem Sinne entspricht der Extremsport mehr dem Verlan-
} 
mitivism und der New Barbarians ${ }^{18}$ zum Ausdruck kam. Die Gewalt wurde nämlich als eine der wenigen Möglichkeiten verstanden, die Grenzen der medial entstellten Welt zu transzendieren. So wurde z.B. die Lebensgefahr beim Bungeespringen zum Prüfstein der Existenz und der Massenmörder zu einem ontologische Fragen stellenden Helden eines Actionfilms. ${ }^{19}$ Die Gewalt gewinnt auf diese Art und Weise epistemologische Züge. Sie erlaubt nicht nur die wahre, d.h. nicht medial vermittelte, Erkenntnis über die einen umgebende Welt, sondern sie ermöglicht vor allem eine tiefgreifende Introspektion, die bei der Suche nach der eigenen Identität helfen soll.

\section{Thomas Hettches Gruselmärchen über die Sehnsucht nach Transzendenz}

Den Zeitgeist der Epoche versucht Thomas Hettche in seinem Roman Nox widerzuspiegeln. Der als einer der ersten Wenderomane angesehene Text bringt zur Sprache und verbindet zwei Übergänge - den einen, regionalen, vom geteilten zum wiedervereinigten Deutschland und den anderen, alle Wirklichkeitsbereiche übergreifenden, von der späten Moderne zur flüssigen Postmoderne diese. Die Handlung des 1995 erschienenen Romans spielt in der Nacht des Mauerfalls, die Ereignisse der Wende treten jedoch in den Hintergrund. Erzählt wird die Geschichte einer jungen Frau, die einem Schriftsteller nach einer Lesung in Westberlin die Frage stellt, ob er jemandem auf dieselbe Art und Weise Leid zufügen könnte, wie er das in seinen Texten dargestellt hat. Später ermordet sie den Mann in ihrer Wohnung und verlässt diese dann, um in der Stadt herumzuirren. Zufälligerweise tut sie das in jener Nacht, in der die Berliner Mauer gefallen ist. Sie lässt sich wahllos auf sexuelle, manchmal sadomasochistisch gefärbte Kontakte ein, um sich schließlich - am Kulminationspunkt des Romans - dem Pathologen Matern und seinen Bekannten als Objekt für ein bizarres sexuelles Ritual hinzugeben, alles mit dem Ziel, sich wieder an ihren Namen zu erinnern, den sie nach dem Mord vergessen hatte. Nachdem sie sich bei der Orgie beinahe ermorden ließ, erfährt sie von jenem Hund, der sie von dem Mord an begleitet hat, ihren wirklichen Namen. Dann geht sie in ihre Wohnung zurück und trifft auf ihr eigenes Mordopfer. Dabei wird die Geschichte die ganze Zeit von ihm,

gen nach Fitness als dem nach Gesundheit, denn er dient in erster Linie dazu, das Erleben von extremen Erfahrungen zu ermöglichen. Die Pflege der Gesundheit und der körperlichen Kraft ist nicht das Hauptziel des Extremsportes, vielmehr kann der Extremsport durch seinen hohen Risikofaktor einen negativen Einfluss auf die Gesundheit des ihn betreibenden Sportlers nehmen. Dazu siehe zum Beispiel KarlHeinrich Bette: X-treme: Zur Soziologie des Abenteuer- und Risikosports, Bielefeld 2004.

${ }^{18}$ Marcus Stiglegger: Das Leben ist Schmerz. Modern Primitivism auf der Suche nach einer neuen Authentizität, in: Testcard, Nr. 8, Mainz 2000, S. 166-177.

${ }^{19}$ Vgl. Tom Holert, Mark Terkessidis: Entsichert - Krieg als Massenkultur im 21. Jahrhundert, Köln 2002, S. 69-116. 
von diesem zwischenzeitlich von der Hauptprotagonistin ermordeten Schriftsteller erzählt. Die konkreten, historischen Geschehnisse und die gesellschaftliche Stimmung werden zwar, einschließlich Nachrichten von den Aktienmärkten ${ }^{20}$, ziemlich genau beschrieben, aber man kann die Tatsache nicht übersehen, dass die Wende in diesem Text eigentlich nur zur Kulisse wird, zum Vorwand, um eine andere Problematik zur Sprache zu bringen. Der Text besitzt ein enormes allegorisches Potenzial. Wofür die Allegorie stehen soll, ist aber nicht selbstverständlich. Hettche bedient sich der oft strapazierten Metapher der Wunde, die scheinbar eindeutig als Symbol für die Berliner Mauer zu interpretieren ist, die einer Wunde gleich, im lebenden Organismus Deutschland aufklaffte. So reflektiert der (tote) Erzähler über die Stadt und die Unruhe der historischen Nacht:

Und ich hörte, wie die Stadt träumte, und wie die Öffnung der Grenze, die sie durchlief wie ihr steinernes Rückgrat, sie ganz langsam erreichte in ihrem Schlaf. [...]

Der Schmerz brannte im Körper der Stadt, und ihre Augen zuckten hinter den geschlossenen Lidern im Schlaf, während das Schiff langsam immer weiter in sie hineinglitt. [...]

Der Lärm widerhallte in meinem Kopf, und ich sah, wie die Stadt um mich erwachte. Während die Vor- und Trabantenstädte noch im Schlaf lagen wie reglose Glieder, begannen die innersten Organe ihres Körpers sich langsam zu bewegen und taumelnd Worte und Bilder durch den Organismus zu pumpen. Es sickerte in sie hinein, was geschah, und überall in den Straßen und Häusern begann die Stadt darauf zu reagieren mit dem Gedächtnis ihrer Leitungen und Kameras, und sie beobachtete und verfolgte, wie das Unvorgesehene immer tiefer in sie eindrang. ${ }^{21}$

Wenn man dazu die sich an Platons Symposion anlehnende Geschichte von den Kugelmenschen in Betracht zieht, drängt sich die wendebezogene Interpretation des Romans sofort auf. An Platons Parabel erinnert sich der Hund zurück, als er im Gespräch mit dem wahrscheinlich als Geist erscheinenden Erzähler Geschichten nennt, die er während seines Dienstes an der Grenze gehört hat. Die Erwähnung des Mythos von den Kugelmenschen könnte an dieser Stelle pathetisch wirken. Wenn man auch auf den nicht besonders einfallsreichen Vergleich der Parabel mit der damaligen politischen Lage Deutschlands und auf die banale Definition von Liebe ${ }^{22}$ Rücksicht nimmt, müsste man zugeben, dass Hettches Text Gefahr läuft, sich in eine kitschige und triviale Wendegeschichte zu verwandeln, die vielleicht um der Auflagenhöhe willen mit pornographischen Beschreibungen ausgestattet wurde. In diesem Sinne wäre Nox als ein Wenderoman ${ }^{23}$ zu verstehen, mit Rücksicht auf die im Text

\footnotetext{
${ }^{20}$ Thomas Hettche: Nox, Frankfurt/Main 1995, S. 31-32.

${ }^{21}$ Ebd., S. 80-82.

${ }^{22}$ Als die Sehnsucht eines Kugelmenschen nach seiner abgetrennten Hälfte definiert.

${ }^{23}$ Der Begriff „Wenderoman“ ist an sich nicht unumstritten. Seine einzelnen Aspekte zu erörtern, würde jedoch den Rahmen dieses Beitrags sprengen. Verwiesen wird hier nur auf die Arbeit von Frank Thomas Grub, die ein ausführliches Kompendium der Wendeliteratur darstellt. Grub hat fünf Aspekte formuliert, die für die Bestimmung, ob ein Text zu der Wendeliteratur gehört, entscheidend sind. Ein Text ist als Wenderoman zu bezeichnen, wenn er sich thematisch-stofflich auf die Wende bezieht und/oder erst nach dem Wegfall der Publikationsbeschränkungen erscheinen darf und/oder das Leben in
} 
omnipräsente Gewalt- und Lustästhetik müsste auch die Einschränkung hinzugefügt werden, dass es sich hier um eine trivialisierte Version oder gar um einen PopWenderoman handelt. Über den Mythos von den Kugelmenschen erzählt der Hund folgendermaßen:

Die Götter, die ihnen ihre vollkommene Form neideten, zerschnitten eines Tages die Kugelwesen. Lange Zeit taten sie daraufhin nichts, als ihre abgetrennte Hälfte zu umarmen. Viele starben vor Hunger und Traurigkeit, bis die Götter sich ihrer erbarmten. Der einen Hälfte der verwundeten Wiesen stülpten sie das Geschlecht nach innen in den Körper hinein. [...]

Ihre wahnsinnige Sehnsucht verwandelte und linderte sich in das, was ihr Liebe nennt. Den nicht endenden Versuch, die Wunde zu heilen, sagte der Hund. ${ }^{24}$

Die Wundemetaphorik der Schlussszene ist sehr bildhaft, aber sie besitzt auch ein hohes Kitschpotenzial und wäre tatsächlich als billig und trivial abzuwerten, wenn nur der Hund vor seinen Erzählungen nicht eine Voraussetzung gemacht hat, die die gesamte wendebezogene Aussage des Textes in Frage stellt. ${ }^{25}$ Die von dem Hund erzählte Geschichte ist nur eine unter vielen möglichen Geschichten. Die historischen Ereignisse bilden nur den Hintergrund für die Geschichten, die später erzählt werden. In der Tat sind die Fakten von geringer Bedeutung, erst die Geschichten bestimmen das, was sich ereignet hat. Es kann sogar davon erzählt werden, was es nie gegeben hat. Diese bedeutsame Feststellung trifft der Hund im Gespräch mit dem Erzähler:

Wir alle drei, sagte er, du und ich und sie, gehören zu einer Geschichte. Zu einer alten Geschichte, die sich wieder ereignet. Warum? Wer weiß? Nichts von dem, was du kennst, wird nach dieser Nacht bleiben, wie es ist. Und nur die Geschichten, die man sich davon erzählt, bestimmen, was wird. Zum Beispiel?

Zum Beispiel finden sich seit 1798, als James Rennell sie kartographierte, auf allen Landkarten Westafrikas die Kong-Berge. Die Legende berichtet, ihre Gipfel seien von ewigem Eis gekrönt. Doch niemand hat sie je bestiegen. Verstehst du?

Wieder schüttelte ich den Kopf.

Es gab sie nie! Der Hund lachte wieder, und er schien sich an seinem eigenen knurrenden Lachen zu verschlucken wie an einem Husten. ${ }^{26}$

Deutschland vor und nach der Wende aus der Perspektive der Nachwendezeit reflektiert und/oder als ein Zeitdokument zu verstehen ist und/oder die Missstände in der DDR thematisiert, die als ein Anzeichen für die Wende betrachtet werden können. Frank Thomas Grub: „Wende“ und „Einheit“ im Spiegel der deutschsprachigen Literatur, Berlin 2003, S. 71-83. Aus dieser Sicht könnte Nox als ein Wenderoman bezeichnet werden, der dem ersten der genannten Aspekten entspricht, wobei die Ereignisse der Wende nur die historische Handlungszeit, aber keine Voraussetzung für die Handlung bilden. Die Geschichte der Mörderin spielt gewissermaßen an den Geschehnissen der historischen Nacht, die aber nicht ausschlaggebend für die Handlungen der Protagonisten sind, vorbei.

${ }^{24}$ Hettche: Nox, S. 158-159.

${ }^{25}$ Vgl. Alban Nikolai Herbst: Einführungstext zu Thomas Hettches Nox-Lesung, http://www.diedschungel.de/ANH/txt/pdf/hettche_nox.pdf, S. 6 [20.06.2013].

${ }^{26}$ Hettche: Nox, S. 156. 
In dem Text wird also eine Geschichte von der Nacht des Mauerfalls erzählt, die die Wunde der Grenze wieder aufgerissen hat. Zugleich wird diese Narration jedoch in Frage gestellt, weil sie eben nur als eine Geschichte relativiert wird, d.h. nur als eine unter vielen möglichen Geschichten. Dabei ist es noch ungewiss, ob gerade diese Erzählung zu der Geschichte wird, die später bestimmen wird, was sich eigentlich in dieser Nacht ereignet hat. Es scheint, als wollte der Autor durch eine auffallend deutliche und suggestive Metaphorik die Leser in die Irre führen, insbesondere diejenigen, die Nox bereits vor der Lektüre als einen politischen oder sogar Wenderoman klassifiziert haben. Die Metapher der Wunde und die Anlehnung an Platons Kugelmenschenmythos kommen der Erwartungen nach dem Roman zur deutschen Einheit ideal nach. Diese Interpretation wird jedoch in dem Text explizit in Zweifel gezogen, in dem angeführten Gespräch des Erzählers mit dem Hundeschutzengel seiner Mörderin. Andere Passagen, in denen die Metaphorik der Wunde auftaucht, weisen auch auf eine andere Geschichte hin, als diejenige, die sich auf den ersten Blick aufdrängt. Ein weiterer Protagonist des Romans, der Pathologe Prof. Carl Matern, bezieht sich in seinen Überlegungen über das in den Verwirrungen der Nacht versunkenen Berlin auch auf die Bilder eines Körpers. Dabei macht er eine Bemerkung, die im Kontext der im Roman omnipräsenten Körper- und Gewaltästhetik besonders schwerwiegend ist:

Die Straße, die zur Grenze führte und jenseits von ihr über die schmale Brücke hinein in den Westteil der Stadt, war für gewöhnlich nachts still und dunkel. Nun wurde sie von den Scheinwerfern der Wagenkolonne erhellt, die an der Charité vorbeirollte. Die Mauer war der Schnitt, mit dem sich die Stadt vom Osten trennte. Wie man ein Glied amputiert, bevor die Ptomaine den ganzen Körper überschwemmen. Wie sehr doch alle Angst hatten vor dem Schmerz, dachte Matern. ${ }^{27}$

Zunächst könnte man diese Stelle als ein weiteres Beispiel der Darstellung von Berlin als einen lebendigen Organismus betrachten, doch bei genauerem Hinsehen kann man einige, auffallende Details bemerken, die den ersten Eindruck sprengen. Zum einen kehrt der Vergleich mit einer Amputation die historische Wahrheit um ${ }^{28}$. Nach Matern diente die Mauer der Abgrenzung Westberlins von dem östlichen Teil der Stadt, der wie ein gangränöses Glied amputiert werden musste. Somit wird Westberlin zum ganzen „Körper“ der Stadt, Ostberlin dagegen nur zu ihrem missgebildeten Teil. Zum anderen befürchtet Matern das Eindringen der Ptomeine, also Leichengifte, vor denen die Grenze zwischen den zwei Teilen Berlins schützen sollte. Das paradoxe Bild passt zu der floskelhaften Symbolik der Wunde nicht besonders gut. Auffällig ist Materns Schlussbemerkung über die Angst vor Schmerz, die dem Bau der Berliner Mauer zugrunde liegen sollte. In diesem Sinne müsste die

\footnotetext{
${ }^{27}$ Hettche: Nox, S. 86-87.

${ }^{28}$ Vgl. Wolfgang Gabler: Der Wenderoman. Zur endlosen Geschichte eines literarischen Genres, in: Kulturnation.de, URL: http://www.kulturation.de/_bilder/pdfs/2012-04-05_Wenderoman.pdf, S. 6 [20.06.2013].
} 
Furcht vor dem Schmerz mit dem Fall der Mauer auch nachlassen, vielmehr würde der Schmerz etwas Erwünschtes bilden, vor dem man sich nicht unbedingt schützen will. Anders formuliert - der Mauerfall und weiter die Wende scheinen mit einer diffusen Erwartung der Radikalität einherzugehen, die sich in Form von Schmerz und schließlich auch Gewalt materialisieren kann. Die Hauptprotagonistin und Mörderin des Erzählers kann tatsächlich eine angespannte Atmosphäre im Europa-Center verspüren, als sie in einer Telefonzelle mit David, einem neulich kennengelernten Sexualpartner, telefoniert und dabei ihre Umgebung aus dem Augenwinkel beobachtet:

Sie öffnete die Augen. Zwei Polizisten patrouillierten so langsam vorüber, als gäben sie sich Mühe, leise zu sein. Vor den Schaufenstern, in den Versorgungsgängen und überall, wo eine Nische war und etwas weniger Licht, hatten sich Menschen hingekauert. Nicht alle schliefen. Viele lagen einfach nur reglos da mit offenen Augen und ohne Blick. Vor sich Flaschen und die Verpackungen der Imbisse. Andere hatten sich die Mäntel über das Gesicht und die Schultern gezogen, Paare lagen eng aneinander, die Beine verschlungen, Familien Kopf an Kopf. Wie von einer Katastrophe hingestreckt, dachte sie. ${ }^{29}$

In dem Einkaufszentrum herrscht eine Stimmung wie nach einer Katastrophe, die Menschen erwarten nach der Maueröffnung nicht nur Unheil, sondern sie scheinen sich auch nach einem Ausnahmezustand zu sehnen. Die Gewalt eines alles umstürzenden Ereignisses ermöglicht die Transgression über die Grenzen der Alltagserfahrung hinweg. Zugleich gibt sie den Betroffenen Einsicht in die wahren Verhältnisse in der Gesellschaft, und in die Eigenschaften, die sowohl der Gesellschaft als auch der eigenen Identität zugrunde liegen. ${ }^{30}$ Auf diese Art und Weise bildet die Gewaltorgie, auf die sich die Hauptprotagonistin einlässt, die Suche nach der fehlenden Katastrophe, die alles Unwichtige außer Kraft setzt, den Einblick in das Wesen der Dinge gewährt und ihnen ihren wahren Namen wiedergibt. Der Gewalt wird hier also eine transzendente Funktion beigemessen, sie soll einen Weg zu der wahren Erkenntnis ermöglichen. Dabei verübt die Mörderin Gewalt und erleidet sie gewissermaßen in Vertretung für die ganze Gesellschaft. Nox erzählt von der Gewalt der Nacht, die es nie gegeben hat, von einem fehlenden Initiationsritus, der die Grenzüberschreitung markiert. So treten die beiden historischen Übergänge, von denen im Roman die Rede ist, nämlich die Wende und die Ankunft der flüchtigen Postmoderne, in den Hintergrund, sie werden nur zu parabelhaften Geschichten. Viel bedeutender scheint die Gewalt zu sein, oder, genauer formuliert, das Gewaltpotenzial, das sich während der revolutionär aufgeladenen Ereignisse gesammelt hat,

\footnotetext{
${ }^{29}$ Hettche: Nox, S. 111.

${ }^{30}$ Die österreichische Schriftstellerin Kathrin Röggla führt in ihrem Essay zum Katastrophenfilm die Faszination, die von einer Katastrophe ausgeht, auf die Sehnsucht nach einer kathartischen Erfahrung zurück. Die Katastrophenbegeisterung sei von dem Verlangen geprägt, im Ausnahmezustand die bestehende Ordnung zugleich negiert und zum Äußersten getrieben zu sehen. Kathrin Röggla: Disaster awareness fair. Zum Katastrophischen in Stadt, Land und Film, Graz 2006, S. 7-30.
} 
jedoch nicht ausgelöst wurde. Die Gewalt, die hier auftaucht, trägt die Maske der Transzendenz, verspricht eine Grenzüberschreitung, aber es ist fraglich, ob sie eine andere, selbstständige Form der Gewalt, eine transgressive Gewalt, oder nur einen Legitimationsversuch für Aggressionstriebe bildet, die sich in der besonderen Konstellation der Postmoderne entwickelt haben.

\section{Der Urknall oder die transgressive Kraft der Gewalt}

Nach Jan Philipp Reemtsma ist Gewalt hauptsächlich auf ein physisches Vorgehen, auf den Übergriff auf den Körper des Anderen ohne dessen Zustimmung zurückzuführen. ${ }^{31}$ In seinem Werk Vertrauen und Gewalt schlägt der deutsche Literaturwissenschaftler eine phänomenologische Herangehensweise an das Phänomen der Gewalt vor, nämlich die Untersuchung der Gewalt nach ihrem Körperbezug. So unterscheidet Reemtsma zwischen drei Formen physischer Gewalt - der lozierenden, raptiven und autotelischen Gewalt. Lozierende Gewalt behandle den Körper des Anderen als Masse, der ein Ort zugewiesen wird. Sie betrachte den Körper des Anderen als ein Hindernis und setze ihn in Bewegung, verschiebe ihn an einen anderen Ort, wo er nicht mehr ein Hindernis darstellt. ${ }^{32}$ Die raptive Gewalt benutze den Körper, um an ihm irgendwelche, meist sexuelle Handlungen zu vollziehen, sie sehe den Körper vor allem als Objekt des Begehrens. ${ }^{33}$ Die autotelische Gewalt könne als das L'art pour l'art der Gewalt verstanden werden, eine Gewalt an sich und um sich selber willen. ${ }^{34}$

Die Feststellung, zu welchem Gewalttyp die in Hettches Text dargestellten Brutalitäten zuzurechnen sind, ist zunächst nicht unproblematisch. Der Roman Nox hat Gewalt nicht nur zum Inhalt, sondern er stützt sich auf Gewalt, deren Verüben die Romanhandlung eigentlich erst ermöglicht. Ein Gewaltakt - die Ermordung des die Handlung erzählenden Schriftstellers - bildet die Voraussetzung für die weitere Narration und verleiht dem Erzähler einen speziellen Status, der sonst nicht zu erreichen wäre, nämlich des auktorialen Ich-Erzählers, der jedoch zugleich als eine eigenständige Person die von ihm erzählten Geschehnisse subjektiv wahrnimmt und einschätzt. Er bleibt dabei kein sich erinnerndes Ich-Erzähler, abgesehen von einigen Rückblenden berichtet er über die Handlung in Echtzeit. Narratologisch gesehen könnte er wegen seines schwebenden ontologischen Status als ein pseudohomodiegetischer Erzähler bezeichnet werden. Diese Lage wäre ohne den Gewaltakt unmöglich. Wäre der Erzähler am Anfang des Textes nicht ermordet worden, könnte er über die Grenzen der dargestellten Welt nicht transzendieren und somit könnte er

\footnotetext{
${ }^{31}$ Reemtsma: Vertrauen und Gewalt, S. 104.

${ }^{32}$ Ebd., S. 108-112.

${ }^{33}$ Ebd., S. 113-116.

${ }^{34}$ Ebd., S. 116-124.
} 
über das für die Führung der Narration notwendige Wissen nicht verfügen. Wäre er aber vom Anfang an als ein allwissender, heterodiegetischer Erzähler konzipiert, könnte er seinen subjektiven und individualisierenden Blick nicht behalten, der zusätzlich durch Verwendung mehrerer Paralipsen unterstrichen wird. Diese zwiespältige Natur des Erzählers kommt zum Ausdruck, als er über die Suche der Mörderin nach ihrem Namen berichtet:

Sie spürte noch einmal das Messer in ihrer Hand. Kühl und angenehm schwer. Wie es meine Haut traf. Den weichen Widerstand des Gewebes. Hörte den Ton, als ich durch die Wunde zu sprechen versuchte. Ein einzelnes Wort. Versuchte sich zu erinnern. Ihren Namen, wußte sie, kannte ich nicht. Und in diesem Augenblick vergaß sie ihn selbst. ${ }^{35}$

Mit dem Ich des Erzählers, das den Namen der Mörderin nicht kennt, meint er seine in einem Zimmer der Wohnung von der Mörderin liegende und noch nicht völlig kalt gewordene Leiche. Er spricht hier über sich selbst als eine Figur, denn als Erzähler gelangte er mit seinem Tod, d.h. mit der Erreichung der Transzendenz, zur Allwissenheit, wovon er oft Gebrauch macht, indem er zufällige detaillierte Daten angibt oder über Ereignisse berichtet, die in weit entfernten Teilen des Landes geschehen sind:

\begin{abstract}
Ich wußte, an den Aktienmärkten war es zu einer deutlichen Erholung gekommen. Durch die von den USA ausgehenden Zinssenkungstendenzen und den stabilisierten Rentenmarkt waren vor allem Bauwerte, aber auch Papiere aus dem Versorgungsbereich und Kaufhaustitel gefragt. Diskontsatz und Lombardsatz weiterhin unverändert. Ich kannte Dollar- und Goldkurs und wußte, daß die Menge der im Westen kursierenden Ostmark, die seit dem Sommer immer weiter angestiegen war, zehn Millionen erreichte, während sie noch immer schlief und zu ihren Füßen der Hund. Im Ostteil der Stadt berief die SED zum vierten Mal in ihrer Geschichte eine Parteikonferenz ein, und weit im Süden, an der Germaniastraße, erlitt ein Mädchen schwere innere Verletzungen als es von einem Lastwagen erfaßt wurde. ${ }^{36}$
\end{abstract}

Zugleich enthält er mehrere Informationen vor, von denen er zu Lebzeiten nicht Bescheid wissen konnte, wie der Name der Mörderin, die er aber mit seinem posthumen Transzendieren erfahren hat. Dabei verstärken sowohl das Spiel des Erzählers mit seiner neu erworbenen Allwissenheit, als auch die ontologische Unmöglichkeit eines toten Ich-Erzählers, den Eindruck der Fiktionalität, was auch inhaltlich in dem Gespräch zwischen dem Erzähler und dem Hund und in der Kong-BergeGeschichte des Letzteren zur Sprache gebracht wird.

Für den Erzähler wirkte also der Gewaltakt transgressiv, gar transzendent, denn er ermöglichte ihm die Überschreitung der Grenzen von der dargestellten Welt. Er reflektiert selbst darüber, als er feststellt, dass man sich von den Dingen paradoxerweise erst befreien kann, nachdem man selbst zum Ding wird:

\footnotetext{
${ }^{35}$ Hettche: Nox, S. 28.

${ }^{36}$ Ebd., S. 31.
} 


\begin{abstract}
Alles, was geschieht, nistet an den Dingen, oxidiert und überzieht sie mit einem unsichtbaren Schmelz. Man sieht das nicht. Der Körper, in dem wir sind, tut nur so, als gehorchte er uns. Doch er allein entscheidet, wohin er den Blick wendet, und wir bemerken nicht, daß wir durch seine gleichgültigen Augen über die Dinge huschen, wie er will. Nur, wenn man tot ist, hört man, wie in einer Stadt alles die Steine zerfrißt. Nun den Dingen gleich, öffnete die Stadt sich hinein in meinen Kopf, und mein Körper reflektierte ihren Lärm. ${ }^{37}$
\end{abstract}

Diese Verdinglichung geht mit der Allwissenheit, mit dem endgültigen Überblick über die Dinge einher. Die dem Körper angetane Gewalt soll dementsprechend zur Gewalt über den Körper führen. Zumindest hat sie in Bezug auf den Erzähler solch eine Wirkung, jedoch es bleibt nur eine Spekulation, ob die Mörderin die Gewalt mit solcher Absicht, also als eine transgressive Gewalt ausgeübt hat. Ihre Tat könnte nach der phänomenologischen Einteilung von Jan Philipp Reemtsma nur als autotelische Gewalt klassifiziert werden. Die Ermordung des Schriftstellers war bestimmt kein Akt der lozierenden Gewalt. Er bildete kein Hindernis, das aus dem Weg geräumt werden müsste, damit die Frau ein bestimmtes Ziel erreichen könnte. Mit hoher Wahrscheinlichkeit lässt sich auch der raptive Aspekt des Mordes ausschließen. Dass die Protagonistin den Schriftsteller ermordet hat, um ihre sexuelle Übermacht zu genießen, bleibt zweifelhaft, weil sie nach der Tat alles andere als sexuell befriedigt zu sein scheint. Obwohl die sexuelle Befriedigung durch Benutzung des Körpers des Anderen in den meisten Fällen das Ziel der raptiven Gewalt sei, sei die Befriedigung des Gewalttäters für die Klassifizierung als raptive Gewalt ohne Belang. Wichtig sei nur die Inbesitznahme des Körpers, raptive Gewalt wolle den Körper haben. ${ }^{38}$ Solcher Beweggrund trifft in diesem Fall auch nicht zu. Die Tat der Protagonistin scheint nur eines zum Ziel zu haben - die Zerstörung des Körpers. Sie tötet den Schriftsteller nur um ihn zu töten, der Mord ergibt sich nicht zufälligerweise bei einem Überfall, einer Geiselnahme, Vergewaltigung o. a. Der Tod des Schriftstellers wird zum Selbstzweck des ihm von der Protagonistin zugefügten Schadens. Zumindest kann man diesen Eindruck von der emotionslosen und mit medizinischer Detailliertheit geschilderten Szene des Mordes gewinnen:

Wie ein Arzt, der die Arterie sucht und den Pulsschlag, legte sie Zeigefinger und Mittelfinger an meinen Hals. Ihre Berührung war sanft und ohne Hast. Doch bevor ich die Augen schloß, sah ich auf ihrem nackten Unterarm wie Firnis Schweiß. Saß blicklos lange unter ihr und rieb, den Kopf weit im Nacken, meine Stirn an ihrem Schoß. Erst, als sie ihre Hand plötzlich wegnahm und die andere hochriß, öffnete ich die Augen. Da sah ich das Messer. Und sie durchschnitt mir die Kehle. Von links nach rechts schnitt sie, und die scharfrandige Wunde klaffte sofort weit auf. Tief schnitt sie in Muskeln und Fleisch, trennte den Kehldeckel vom Kehlkopf, durchschnitt Halsschlagader und Schilddrüsenschlagader, kappte mir Luftröhre und Speiseröhre, und schnitt tief noch in einen Halswirbel hinein. Als sie das Messer aus der Wunde nahm, zog sie keinen Menschenlaut, doch mehr als ein gurgelndes Geräusch, mit heraus. [...]

\footnotetext{
${ }^{37}$ Hettche: Nox, S. 30-31.

${ }^{38}$ Reemtsma: Vertrauen und Gewalt, S. 113.
} 
Noch eine ganze Weile hielt sie das Messer über mir. Schwer und angenehm fühlte sie es in ihrer Hand. Da war ich längst tot. Der nasse Vorhang bauschte sich und schlug gegen das Fenster. Reglos stand sie da und lauschte dem Wind nach. Dann ließ sie das Messer fallen und ging. ${ }^{39}$

Die in dieser Szene dargestellte Gewalt lässt sich auf der phänomenologischen Ebene des Körperbezugs eindeutig als autotelische Gewalt betrachten. Die wahren Motive der Protagonistin bleiben unklar, der Mord an dem Erzähler verläuft selbst so leidenschaftslos und gleichgültig, dass er einer Hinrichtung, oder, was in diesem Kontext besonders gewichtige Assoziationen auslösen lässt, einem Ritual ähnelt.

\section{Hic nox, hic salta}

Interessanterweise empfindet die Mörderin nach ihrer Tat auch ein gewisses Gefühl der Verdinglichung, wobei es aber in ihrem Fall eine Reduzierung der Wahrnehmung zur Folge hat. Sie verliert sich in den Gewalttaten bis zu diesem Grad, dass sie den eigenen Namen vergisst. Die gegen den Körper des Anderen angewandte Gewalt übt eine bestimmte Wirkung auch auf den Körper der Protagonistin aus. Die „transgressive“ Gewalt scheint sich auf eine Dualität zu stützen, der Gewalttäter ist nicht nur dazu bereit, dem Anderen Schaden zuzufügen, sondern er nimmt auch in Kauf oder zielt sogar unmittelbar darauf ab, dass er der Gefahr, selbst Gewaltopfer zu werden, ausgesetzt sein wird. Auf diese Art und Weise ähnelt die Gewalt einem Übergangsritus, der nach dem französischen Anthropologen Arnold van Gennep aus drei Phasen bestehe, nämlich der Ablösungs-, Zwischen- und Angliederungsphase. ${ }^{40}$ Der Ritualmord an dem Schriftsteller hat die Protagonistin von der Gesellschaft abgelöst, indem er ihre soziale Position zu der einer Mörderin auf der Flucht reduziert hat. Symbolisch hat sich dieser Wandel durch das Vergessen des Namens geäußert. Der Ablösung von der Gesellschaft folgte die Trennung von der eigenen Identität, nicht nur ihr Name, sondern auch ihr Gedächtnis, ihre Erinnerungen an die eigene Person sind in Vergessenheit geraten:

Es war, als entkleidete sich beim Gehen ihr Gedächtnis mit jedem Schritt. Verschwunden schon die eigene Geschichte. Wie fremde Photographien blitzten Erinnerungsbilder auf, die sie, gleichgültig, nicht mehr ordnete. ${ }^{41}$

Der Verlust der eigenen Identität geht mit dem Prozess der Verdinglichung einher, die Protagonistin hört auf, ein Gewalt verübendes Subjekt zu sein, und wird zum Objekt, dem Gewalt zugefügt wird. Die Reduzierung auf den Körper kennzeichnet die Zwischenphase des Rituals und bildet die Grundlage für den (Wieder)Aufbau der eigenen Persönlichkeit. Die beinahe atavistische Rückkehr zur Körperlichkeit setzt

\footnotetext{
${ }^{39}$ Hettche: Nox, S. 10-11.

${ }^{40}$ Arnold van Gennep: Übergangsriten, Frankfurt/Main 1986, S. 21.

${ }^{41}$ Hettche: Nox, S. 27.
} 
in der flüchtigen Welt feste, unüberschreitbare Grenzen - die Grenzen des eigenen Körpers. Für die Protagonistin bildet ihr Körper zunächst die einzige Instanz, auf die sie sich verlassen und der sie trauen kann:

Der Hund zu ihren Füßen stellte ein Ohr am noch reglosen Körper. [...]

Das Licht der Scheinwerfer stach jetzt in den Himmel und umgab das hohe Gras mit unzähligen Schlagschatten. Sirren stand um die Lampen in der Luft. Sie dachte, daß sie noch immer ihren Namen nicht mehr wußte. Wenig mehr von sich als das, was ihre Haut registrierte. Die Kälte und die Weise, wie sie atmete und wie die Muskeln sich spannten. War ein Tier neben dem Tier, das neben ihr lag. So blieb ihr nichts, sie stand auf und ging los. Jenen nach, die vor ihr im dämmrigen Nachmittag verschwunden waren. ${ }^{42}$

Dabei übernimmt der Körper die Rolle des unfehlbaren Wahrnehmungsapparates. Diese Perspektive steht im krassen Widerspruch zu der nur durch Medien verschiedenster Art vermittelten Wirklichkeit, der Körper ermöglicht somit die einzige authentische und unmittelbare Erfahrung. Die Loslösung von ihrer Identität und den eigenen Erinnerungen erweckt bei der Protagonistin ein Gefühl der Selbstentfremdung, die neu gewonnene Körperlichkeit macht auf sie einen Eindruck der Fremdheit:

Wieder das Geräusch ihrer Schritte, auf das sie, vom Hermannplatz zum Landwehrkanal, mit einem Mal begonnen hatte zu achten. Zuerst war ihr das klackende Geräusch der Absätze und das hell nachgängige Tickern der Ledersohlen auf den Steinplatten der Bürgersteige fremd gewesen. Es hatte sie gewundert, wie es sich gegen den Lärm der Straßen durchsetzte und vom Ineinander der Geräusche unterschied, stets bei ihr, als fände es sonst keinen Halt.

Der aufgeschlagene Kragen der Lederjacke hatte ihr unter dem Haar im Nacken gerieben. Ob es kalt gewesen war dort im Gras, hatte sie sich gefragt, als wäre ihr Körper ein sehr fernes Gebiet. Die Entfernung nimmt immer noch zu, hatte sie gedacht, und für Momente nicht gewußt, ob die Schritte ihr folgten oder sie den Schritten, die sie nah bei sich hörte. Und so, als folgte sie einer Spur, war sie schließlich zu jener Brücke gekommen. Die Hände auf dem Geländer, hatte sie lange ins Wasser hinabgesehen. Die Möwen, bewegungslos und ohne einen Flügelschlag im Wind, dicht über ihrem Kopf. ${ }^{43}$

Um sich des eigenen Körpers zu vergewissern, wie in psychotischen Zuständen, muss dem Körper Leid zugefügt werden. Der depersonalisierte Körper wird verletzt und durch den Schmerz kommt es zu einer Vereinigung, die Einheit der Person wird wiederhergestellt. ${ }^{44}$ Der Schmerz trägt hier zu einer völligen Reduzierung des Individuums auf ein Bündel einfachster Reflexe und Instinkte bei. ${ }^{45}$ Die dem Körper

${ }^{42}$ Ebd., S. 37.

${ }^{43}$ Ebd., S. 39-40.

${ }^{44}$ Vgl. Franz Resch: Schmerz tut gut - Der Körper als Instrument zur Bewältigung psychischer Krisen, in: Wie viel Körper braucht der Mensch? Standpunkte zur Debatte, hrsg. von Gero von Randow, Hamburg 2001, S. 101-108, hier 106.

${ }^{45}$ Der deutsche Soziologe Wolfgang Sofsky schreibt darüber: „Nirgendwo ist der Mensch mehr Kreatur als im Zustand unerträglicher Schmerzen. [...] Der Schmerz tilgt den Abstand zur Situation und zu sich selbst. Das Selbst wird in die Gegenwart eingeschmolzen. Die Differenz zwischen Außen und Innen, zwischen Geschehnis und Erlebnis ist ausgelöscht. Im Schmerz ist der Mensch ganz Leib, nichts sonst. Die Kontrolle über den Körper ist dahin. Er ist kein Werkzeug des Handelns mehr. Der Schmerz 
angetane Gewalt bringt die ganze Identität der Person auf Null und somit versetzt sie in einen Schwebezustand zwischen der sozialen Ordnung, von der sich die Person in der ersten Phase der Rituals abgelöst hat, und dem neuen gesellschaftlichen Rahmen, in den sie erst nach der Vollendung des Rituals eingegliedert wird. ${ }^{46}$ Die sich in dem Schwebezustand befindende Protagonistin verwickelt sich in zufällige, sexuelle Bekanntschaften, die oft in sadomasochistische Praktiken übergehen. Bedeutsam ist die Tatsache, dass diese Beziehungen höchst depersonalisiert zu sein scheinen und die Protagonistin immer zu einem sexuellen Objekt herabgesetzt wird:

Auf einem Tisch, dachte sie, liegt alles offen, in Reichweite von Hand und Blick. Alle Dinge, dachte sie, besiedeln einmal seine Oberfläche und warten darauf, daß man sie gebraucht. Wie auf eine Lichtung kommen sie zuverlässig hierher. Nur ich, dachte sie, habe keinen Ort und bin weniger als die Dinge.

Sie nahm die beiden Weingläser und stellte sie auf den Nebentisch. Ob sie etwas trinken wolle, fragte die Frau. Sie schüttelte den Kopf. Nahm die Flasche und stellte auch sie weg. Wie sie heiße, fragte die Frau. Sie antwortete nicht. Ich bereite mir, dachte sie, einen Tisch im Angesicht meiner Feinde. Sie strich über die Tischdecke hin. Will mich, dachte sie und mußte lächeln bei dem Gedanken, wie die Dinge niederlassen in ihren Blicken, die sich über mir kreuzen sollen wie über allem anderen.

Sie stand auf, schob den Stuhl zurück und kroch, mit den Händen sich haltend und mit dem linken Knie voran, auf den Tisch. Drehte sich aus der Hocke auf den Rücken. Im Nacken und in den Kniekehlen hart die Kanten des Tisches. Kreuzte die Arme über der Brust und schloß die Augen. Dachte an das Messer und daran, wie die Erinnerung ihr durch den Leib schnitt. Welchen Namen, dachte sie, hat das Ding, das ich bin? ${ }^{47}$

Nachher kommt es zum Geschlechtsverkehr zwischen der Protagonistin und dem im Restaurant eine Weile zuvor kennengelernten Mann. Die Figuren zeigen jedoch weder Lust noch Vergnügen auf, wiederum fällt der rituelle Charakter der Handlungen auf. Die Protagonistin gelangt schließlich nach ihrer Irrfahrt durch Berlin in das berühmte Ost-Berliner Krankenhaus Charité, wo sie in dem „Anatomischen Theater", wie man den Sektionshörsaal bezeichnete, an einem bizarren, orgiastischen Ritual teilnimmt, das zum Kulminationspunkt des Romans wird. Bevor es jedoch dazu kommt, lernt sie Professor Matern, den Leiter des Instituts für Pathologische Anatomie der Charité, kennen, der später die Rolle des Zeremonienmeisters bei der Orgie übernimmt. Bei dem kurzen Gespräch, die die beiden miteinander führen, fallen Worte, die den Interpretationsschlüssel zu den weiteren brutalen Geschehnissen bieten:

entmachtet die Person.“ In der Fußnote zu dieser Passage erwähnt Sofsky Rituale der Initiation und asketische Techniken als Beispiele für kulturelle Praktiken des Schmerzes. Wolfgang Sofsky: Traktat über die Gewalt, Frankfurt/Main 1996, S. 74 und 231.

${ }^{46}$ D.h. in den Zustand der „Liminalität“. Vgl. Victor Turner: The Ritual Process. Structure and Anti-Structure, New Jersey 2008, S. 94-96.

${ }^{47}$ Hettche: Nox, S. 42-43. 
Er hat blaue Augen, dachte sie und bemerkte, wie unter dem hageren Gesicht mit tiefen Falten auf den glattrasierten Wangen spitz der Adamsapfel über den weißen Stehkragen des Arztkittels zuckte. Rotgewaschene Hände. Nervös und unruhig rieben sie sich aneinander, und sein Blick wischte unstet über sie hinweg, so, als hätte man ihn bei etwas unterbrochen und er wüßte nicht, was nun zu tun sei.

Ich bin Professor Matern. Guten Abend. Waren Sie schon mal im Osten?

Sie schüttelte den Kopf.

Hic nox hic salta, sozusagen. Sie sind doch nicht etwa nervös?

Sie verstand nicht. Nox, wußte sie, hieß Nacht. Er zischte das Wort, bis der Ton nur mehr ein scharfer Hauch war. Wie Gas, das aus einer Kartusche entwich. Sein Blick ging ziellos über sie hinweg. Doch plötzlich verharrte er und wurde ruhig. Jetzt sieht er Lara an, dachte sie.

Am lebendigen Leib, verstehen Sie? Sie reißen die Narbe auf, die so gut verheilt schien. In dieser Nacht, verstehen Sie? Man muß neu begrenzen, ins Wuchernde schneiden, tief ins Lebendige hinein.

So, als liefe ein Film weiter, der unbelichtet nur noch Weiß zeigt und sich dennoch schattenhaft auf der Leinwand mit Bewegung füllt, hörte sie ihm zu. ${ }^{48}$

Gewalt wird hier als der Augenblick der Probe verstanden, als Moment der endgültigen Entscheidung, die keine Rückkehr oder nochmaliges Nachdenken zulässt, als Prüfstein der Existenz und der eigenen Möglichkeiten. Materns prägnanter Satz, der als Motto für den ganzen Roman dienen könnte - „Hic nox, hic salta“ - spielt auf den lateinischen, aus einer Fabel von Äsop stammenden Spruch ,hic Rhodus, hic salta“. Die Nacht, die als ein Ausnahmezustand, der die bisher gültigen Grenzen, sowohl die politischen als auch die moralischen, abschafft ${ }^{49}$, wird somit zum Moment der Verifizierung aller früher deklarierten Absichten, in dem der Spruch „Zeig, was du kannst" zum Grundprinzip zwischenmenschlicher Beziehungen wird. Nach dem Gespräch wird die Protagonistin zur Teilnahme an einer seltsamen Folterséance eingeladen. Die nackte Protagonistin wird an einen Sektionstisch in dem ,Anatomischen Theater" angebunden und dann mit einer Winde hochgezogen, wie ein biologisches Präparat, an dem eine Vivisektion durchgeführt werden soll. Tatsächlich greift eine der an der Séance Beteiligten nach chirurgischen Instrumenten, wie Skalpelle und Lanzetten, mit der Absicht, sie an dem lebendigen Körper der Protagonistin zu verwenden, jedoch im letzten Augenblick erscheint der Hund, der im Laufe des Textes Züge eines Schutzengels der Mörderin annimmt. In einer Art Deus ex Machina-Lösung erfährt die Protagonistin wieder ihren Namen, wodurch das Ritual der transgressiven Gewalt zur Vollendung gebracht wird:

\footnotetext{
${ }^{48}$ Ebd., S. $127-128$.

${ }^{49}$ Die Nacht des Mauerfalls in Hettches Text könnte gewissermaßen als die Zeit des Karnevals im Sinne Bachtins verstanden werden. Die Nacht bringt eine vorübergehende Abschaffung der sozialpolitischen Ordnung mit sich, wobei es aber nicht eindeutig ist, ob dieses Fest der kontrollierten Unordnung die Funktion eines Sicherheitsventils erfüllt und der Bestätigung der bisherigen Machtinstanzen dient. Die Beteiligung an den Geschehnissen durch Vertreter des bisherigen Systems, wie den Senatsrat Dr. Ewald Roll, lässt einen die Nacht auf diese Art und Weise interpretieren. Vgl. die Szene des Empfanges der Ostdeutschen während einer westdeutschen Party und die symbolische Unterwerfung durch Oralsex. Hettche: Nox, S. 67-69.
} 
Die Winde zog schnarrend, Zahnrad auf Zahnrad, an, das Seil zitterte, doch kurbelte Matern sie versehentlich nach oben, und plötzlich war sie ganz nah bei David und sein Mund an ihrem Ohr. Denk an mich, flüsterte er, und sie küßte ihn schnell. Noch heute wirst du mit mir im Westen sein. Dann klapperte die Winde, und die Kälte des Metalls verbrannte ihren Rücken. Sie wurde umgedreht, man löste ihre Handfesseln, und der Hund setzte, ohne zu zögern, mit einem Sprung hinter ihr auf den stählernen Tisch.

Sie schrie, versuchte ihm zu entkommen, für einen Moment verloren seine Pfoten unter ihrem Zukken auf dem glatten Metall den Halt, er rutschte weg, jaulte auf, doch fester gruben sich die Krallen erneut in ihre Haut. Speichel tropfte ihr auf den Rücken, und seine Zunge leckte darüber hin. Der Schmerz war ein glänzendes, gläsernes Stückchen Zeit, das sich einbrannte und in ihr zu schwelen begann. Ein Augenblick, in den sie wie in glitzernde Scherben stürzte, und die Wunde verlief durch sie hindurch, die aufgebrochene Wunde. Im Schoße der Jungfrau die geronnenen Gedanken Gottes, dachte sie. Dann war plötzlich die Schnauze des Hundes neben ihrem Kopf, Hecheln ganz dicht an ihrem Ohr, und der Hund flüsterte ihren Namen. ${ }^{50}$

Die Gewalt erreicht ihren Kulminationspunkt, die Protagonistin wird der unbegrenzten Gewalt ausgesetzt, die als autotelische Gewalt betrachtet werden kann, denn die Runde im Anatomischen Theater scheint kein anderes Ziel zu verfolgen, als die Verletzung des Körpers von der Mörderin. Das Sich-Ausliefern der Gewalt kann als eine Art Selbstopferung angesehen werden, deren Ergebnis die Aufnahme in den Westen bilden sollte, wie in den Himmel, wobei der Westen hier nicht nur politisch, sondern auch als die Welt der Postmoderne, mit ihrer flüchtigen Grenzen, in denen der Aufbau der eigenen Persönlichkeit einen nie endenden Prozess bildet, verstanden werden kann. Die Anspielung auf den Bibelvers ${ }^{51}$ hebt wiederum den rituellen Charakter der Gewalttätigkeiten, in die sich die Protagonistin verwickelt, hervor. ${ }^{52}$

\section{Ein neuer Ort für autotelische Gewalt?}

Die den Roman von Thomas Hettche durchdringende Gewalt bildet mehr als eine effektvolle Drastik, die das Kontroverse des Textes ausmachen und somit die Auflagenhöhe vorantreiben soll. Auf Gewalt gründet sich der Text, sie konstituiert auch den ontologischen Status der beiden Hauptprotagonisten. Der Schriftsteller überschreitet durch seinen gewaltsamen Tod die Grenzen der dargestellten Welt und wird somit zum Erzähler des Textes. Für die Mörderin bedeutet jede Gewalttat einen weiteren Schritt zur Selbsterkenntnis, die ritualisierte Gewalt ermöglicht die Transzendierung der eigenen Identität, damit diese aufs Neue aufgebaut werden könnte. In beiden Fällen wird dem Körper durch den ritualisierten Angriff an ihn mehr Bedeutung beigemessen als bloß einem biologischen Organismus. Diese pervertierte Wiederentdeckung des Körpers repräsentiert, sowohl im Kontext der postmodernen und

\footnotetext{
${ }^{50}$ Hettche: Nox, S. 139-140.

${ }^{51}$ „Und Jesus sprach zu ihm: Wahrlich ich sage dir: Heute wirst du mit mir im Paradiese sein.“ Lk 23, 43.

${ }^{52}$ Die Anspielung schafft dabei eine enorme Spannung zwischen dem Transzendenten und Sakralen des Evangeliums und dem Körperlichen, Sexuellen und Gewalttätigen der geschilderten Folterorgie.
} 
globalen als auch der politischen, nur auf Deutschland bezogenen Wende, die Sehnsucht nach einer neuen Authentizität. ${ }^{53}$ In Bezug auf die Entrealisierung der Cyberspace-Epoche bildet die Rückkehr zum physischen, verletzbaren Körper eine Art Selbstbesinnung, den Wiedergewinn von haltbaren Grenzen. ${ }^{54}$ Im Kontext der Wende können die Gewaltphantasien als Spuren der Unzufriedenheit mit dem auf friedlichen Wegen erreichten Elitenwechsel und als eine diffuse Sehnsucht nach einem klaren Einschnitt im Sinne eines Gründungsmordes betrachtet werden. ${ }^{55}$ Unabhängig von der Begründung und dem gesellschaftlichen Hintergrund verwandelt sich die Gewalt als Mittel der möglichen Transgression in einen Lebensstil, die instrumentell verstandene Gewalt, die der Transzendierung dienen sollte, entgleist und wird zum Selbstzweck. Das legt die Vermutung nahe, dass diese transgressive Gewalt aus phänomenologischer Sicht eigentlich als die autotelische Gewalt zu bezeichnen ist, die kein Ziel außer sich selbst hat, obwohl sie instrumentell gedeutet werden kann. Die postmoderne Ritualisierung der Gewalt in Hettches Roman kann also als ein Versuch verstanden werden, einen neuen kulturellen Ort für die autotelische Gewalt zu finden. ${ }^{56}$

\section{Literatur}

Baudrillard, Jean: Simulacres et Simulation, Paris 1981.

Baudrillard, Jean: Amerika, Berlin 2006.

Bauman, Zygmunt: Flaneure, Spieler und Touristen. Essays zu postmodernen Lebensformen, Hamburg 1997.

\footnotetext{
${ }^{53}$ Das Authentische der Körpererfahrung wird zusätzlich durch den Widerspruch zwischen der datenüberladenen Wirklichkeit des (postmodernen) Erzählers, der über viele nicht gleichbedeutende Ereignisse auf die gleiche Art und Weise berichtet und sie damit relativiert, und der von der gehetzten Mörderin wahrgenommenen Wirklichkeit, die nur auf das zum Überleben Notwendigste reduziert wird. Die nur durch körperliche Erfahrung wahrgenommene Welt der Mörderin steht auch im Widerspruch zu der undurchschaubaren Welt der Prominenten, die nach der Wende verschiedene, nicht nur politische Intrigen spinnen.

${ }^{54}$ „Das Spiel mit dem Körper ist [...] vor allem ein Spiel mit dem Schmerz. Die als bedrohlich empfundene Entmenschlichung durch ein Fortschreiten der Technik wird [...] immer mit einer Verdrängung des Menschen in der Welt assoziiert. [...] Um ein neues Bewusstsein für den Körper zu schaffen, wählt der Künstler den Schmerz als stärkste mögliche Körperwahrnehmung, um öffentlich dessen Bedeutung zu proklamieren. Der Mensch ist erst wieder Mensch, wenn er dem Leid in seinem Leben seinen Platz zugesteht.“ Christian Klotz: „Fraktales Fleisch“? Körpermodifikationen und Modern Primitivism als Ausdrucksformen einer neuen (und alten) Körperlichkeit, Dissertation, Marburg 2012, http://archiv.ub.uni-marburg.de/diss/z2012/1051, S. 61-62 [20.06.2013].

${ }^{55}$ Vgl. Philipp Ther: 1989 - eine verhandelte Revolution, in: Docupedia-Zeitgeschichte, 11.2.2010, http://docupedia.de/docupedia/images/4/48/1989.pdf [20.06.2013].

${ }^{56}$ Nach Reemtsma habe die autotelische Gewalt in der atlantischen Kultur der Moderne keinen legitimierbaren Ort mehr. Reemtsma: Vertrauen und Gewalt, S. 123-124. Dass diese Gewaltform aus der Öffentlichkeit verschwunden und nicht mehr geregelt ist, bedeutet jedoch nicht, dass sie nicht mehr ausgeübt wird. Vgl. Sofsky: Traktat über die Gewalt, S. 125.
} 
Bauman, Zygmunt: Flüchtige Moderne, Frankfurt a.M. 2003.

Bessing, Joachim; u.a.: Tristesse Royale: Das popkulturelle Quintett, Berlin 1999.

Bette, Karl-Heinrich: X-treme: Zur Soziologie des Abenteuer- und Risikosports, Bielefeld 2004.

Fukuyama, Francis: The End of History and the Last Man, New York 2006.

Gabler, Wolfgang: Der Wenderoman. Zur endlosen Geschichte eines literarischen Genres, in: Kulturnation.de, URL: http://www.kulturation.de/_bilder/pdfs/2012-04-05_Wenderoman.pdf [20.06.2013].

Gennep, Arnold van: Übergangsriten, Frankfurt a. M. 1986.

Grub, Frank Thomas: „,Wende“ und „Einheit“ im Spiegel der deutschsprachigen Literatur, Berlin 2003.

Heilbroner, Robert: The Triumph of Capitalism, in: The New Yorker, 23.01.1989, S. 98.

Herbst, Alban Nikolai: Einführungstext zu Thomas Hettches Nox-Lesung, http://www.die-dschungel. de/ANH/txt/pdf/hettche_nox.pdf [20.06.2013].

Hettche, Thomas: Nox, Frankfurt a. M. 1995.

Holert, Tom; Terkessidis, Mark: Entsichert - Krieg als Massenkultur im 21. Jahrhundert, Köln 2002.

Jung, Thomas: Trash, Cash oder Chaos? Populäre deutschsprachige Literatur seit der Wende und die sogenannte Popliteratur, in: Alles nur Pop? Anmerkungen zur populären und Pop-Literatur seit 1990, hrsg. von Thomas Jung, Frankfurt a.M. 2002, S. 15-27.

Klotz, Christian: „Fraktales Fleisch“?: Körpermodifikationen und Modern Primitivism als Ausdrucksformen einer neuen (und alten) Körperlichkeit, Dissertation, Marburg 2012, http://archiv.ub.unimarburg.de/diss/z2012/1051 [20.06.2013].

Matussek, Peter: Real Virtuality - Die Wahrheit der Entwirklichung, in: Space and Truth - Raum und Wahrheit, hrsg. von Thea Breszek, Wolfgang Greisenegger, Lawrence Wallen, Zürich 2009, S. 206-215.

McLuhan, Marshall: Understanding Media: The Extensions of Man, Cambridge 1994.

Reemtsma, Jan Philipp: Vertrauen und Gewalt: Versuch über eine besondere Konstellation der Moderne, Hamburg 2008.

Resch, Franz: Schmerz tut gut - Der Körper als Instrument zur Bewältigung psychischer Krisen, in: Wie viel Körper braucht der Mensch? Standpunkte zur Debatte, hrsg. von Gero von Randow, Hamburg 2001, S. 101-108.

Röggla, Kathrin: disaster awareness fair. zum katastrophischen in stadt, land und film, Graz 2006.

Sofsky, Wolfgang: Traktat über die Gewalt, Frankfurt a.M. 1996.

Staffel, Tim: Terrordrom, München 2000.

Staffel, Tim: Rauhfaser, Frankfurt a.M. 2002.

Stiglegger, Marcus: Das Leben ist Schmerz. Modern Primitivism auf der Suche nach einer neuen Authentizität, in: Testcard, Nr. 8, Mainz 2000, S. 166-177.

Ther, Philipp: 1989 - eine verhandelte Revolution, in: Docupedia-Zeitgeschichte, 11.2.2010, http://docu pedia.de/docupedia/images/4/48/1989.pdf [20.06.2013].

Turner, Victor: The Ritual Process. Structure and Anti-Structure, New Jersey 2008.

Virilio, Paul: Krieg und Fernsehen, Frankfurt a.M. 1997.

Wirz, Mario: Umarmungen am Ende der Nacht, Berlin 2004. 